\title{
Efficient Approximation Using Probabilistically Improved Combinatorial Structure of Bernstein'S Polynomial Operator's Weights through the Fusion of Dual-Perspectives
}

\author{
Shanaz A. Wahid (Corresponding author) \\ Dept. of Mathematics \& Statistics, The University of the West Indies \\ Trinidad \& Tobago, West Indies \\ E-mail: shanazw@hotmail.com
}

Received: March 10, 2011 Accepted: March 24, 2011 doi:10.5539/jmr.v3n3p59

\begin{abstract}
A new polynomial approximation operator has been proposed using the weight-functions of the well-known Bernstein's polynomial operator in its probabilistically improved combinatorial structure, achieved through a rather-ingenious 'Fusion' of two dual-perspectives. The new approximation formula has been compared empirically with the simple classical method of polynomial approximation using the well-known "Bernstein Operator" in its primal-modified form. The percentage absolute relative errors for the proposed approximation formula and that with the 'Primal' form of the modified "Bernstein Operator" have been computed for a certain chosen functions and with different number of node-points in the interval of approximation. It has been observed that the proposed approximation formula produces relatively exceedinglysignificantly better results.
\end{abstract}

Keywords: Combinatorial Structure, Dual-Fusion, Percentage Absolute Relative Errors, Polynomial Approximation, Probability, Weight Functions

AMS Classification: 41A10, 41A36, 62F10.

\section{Introduction}

The main fact was the one which motivated us to ponder-and-wonder as to whether or not we would be able to retain the simplicity of "equi-distant" points of nodes in the interval of approximation in our proposed approximation formula, and still aspire successfully for its optimality-in-efficiency, only through the manipulation of the 'weight-function' [Cheney, 1964; Korovkin, 1960]. In fact we were successful! The following section details the 'How' part of it. And the section following that numerically illustrates that the potential of this new/ proposed polynomial approximation operator was so profound that it could be accepted as almost the 'Optimal' one, inasmuch as it excelled over the 'Primal' version of the well-known usual "Bernstein's Polynomial Approximation Operator", besides being exceedingly superior to that! The "Bernstein's Polynomial Approximation Operator" happened to be the foundational structure for the build-up of the proposed one, using weight-functions derived through a probabilistic approach, and a rather-ingenious 'Fusion' of 'dualperspectives'. The details of the construction of the proposed 'Polynomial Approximation Operator' are presented in what follows.

\section{Proposed Dual-Fusion Weighted Approximation Operator}

The approximation problem arises in many contexts of 'Numerical Analysis and Computing'. The 'Quadrature' is one such context, for example. Wierstrass (1885) proved his celebrated approximation theorem: If 'f ' $\epsilon C[a, b]$; for every $\delta>$ 0 , there is a polynomial 'p' such that $|f-p|$, delta. In other words, the result established the existence of an algebraic polynomial, in the relevant variable, capable of approximating the unknown function in that variable, as closely as we please [Sahai, 1984; 2004]! The aforementioned result was a big beginning of the mathematicians' interest in 'Polynomial Approximation' of an unknown function using its values generated experimentally or known otherwise at certain chosen 'nodes'/ 'Knots' of the domain of the relevant variable, as of interest to the scientist concerned. The great Russian mathematician S. N. Bernstein proved the Weirstrass' theorem in such a manner as was very stimulating and interesting in many ways. He first noted a simple but important fact that if the Weirstrass' theorem holds for $C[0,1]$, it also hold for $C[a, b]$ and conversely. Essentially $C[0,1]$ and $C[a, b]$ are identical, for all practical purposes, as they are linearly isometric as normed spaces, order isomorphic as algebras (rings). Most important contribution in the Bernstein's proof of this theorem consisted in the fact that Bernstein actually displayed a sequence of polynomials that approximate a given function $\mathrm{f}(\mathrm{x}) \epsilon C[0,1]$. If $\mathrm{f}(\mathrm{x})$ is any bounded function on $C[0,1]$, the sequence of Bernstein's Polynomial for $\mathrm{f}(\mathrm{x})$ is defined by:

$$
\left(B_{n}(f)\right)(x)=\sum_{k=1}^{k=n} w_{k, n} * f(k / n)
$$

Wherein, $w_{k, n}=\left(\begin{array}{l}n \\ k\end{array}\right) \cdot x^{k} \cdot(1-x)^{n-k}$ are the respective weights for the values ' $f\left(\frac{k}{n}\right)$ ' of the function at the knots " $(k / n)$ " $[k=$ 
$0(1) n]$. Now, we are set to launch the "probabilistic perspective" seminal to the proposition of our novel 'Polynomial Approximation Operator' in the same "Equi-Distant Knots" 'Set-Up' of the Bernstein's Operator, in what ensues. We, without any loss of generality as explained earlier, consider the approximation of an unknown function ' $\mathrm{f}(\mathrm{x})$ '; $x \in \mathrm{C}[0$, 1/2]. For such a case the usual 'Bernstein Polynomial Approximation Operator in (1) boils down to:

$$
\left(B_{n}(f)\right)(x)=\sum_{k=1}^{k=n} w_{k, n} * f\left(\frac{k}{2 * n}\right)
$$

Wherein $w_{k, n}=\left(\begin{array}{l}n \\ k\end{array}\right) *(0.5-x)^{k} *(0.5+x)^{n-k}$ are the respective weights for the values' $f\left(\frac{k}{2 * n}\right)$ ' of the function at the knots “ $\frac{k}{2 * n} "[\mathrm{k}=0$ (1) $\mathrm{n}]$. Incidentally, as we could use a suitable transformation (translation-and-change-of-scale) of the variable " $x$ ", we could assume, without any loss of the generality, that we are interested in the approximation of a bounded function $f \epsilon C[0,1 / 2]$, even if the impugned function could, originally, be rather a bounded one in $\mathrm{C}[\mathrm{a}, \mathrm{b}]$. We visualize that the line $\mathrm{C}[0,1] \mathrm{O} 1$ to $\mathrm{O} 3$ with the point $\mathrm{O} 2$, such that $\mathrm{O} 1$ to $\mathrm{O} 2$ : $\mathrm{O} 2$ to $\mathrm{O} 30 \tilde{0} .5-\mathrm{x}:: 0.5+\mathrm{x} ; \mathrm{x} \epsilon C[0,0.5]$.

In the 'Primal' sense, we could envisage ' $\mathrm{k}$ ' knots sitting on the line from $\mathrm{O} 1$ to $\mathrm{O} 2$, and ' $\mathrm{n}-\mathrm{k}$ ' knots sitting on the line from $\mathrm{O} 2$ to O3. Probabilistically, the probability of this, say 'Primal' possibility could be called the 'Primal ProbabilityWeights'. It would be worth noting in the above that these Primal Probability Weights' " $P_{w 1}(x ; k, n)$ " are the typical coefficients of $\theta^{n}$ in the expansion of the binomial-product $\left[(1+\theta)^{n *(0.5-x)}\right] *\left[(1+\theta)^{n *(0.5+x)}\right]$, i.e.in $(1+\theta)^{n}$.

Therefore, we have:

$$
\sum_{k=0}^{k=n} P_{w 1}(x ; k, n)=\sum_{k=0}^{k=n}\left(\begin{array}{c}
n *(0.5-x) \\
k
\end{array}\right) *\left(\begin{array}{c}
n *(0.5+x) \\
n-k
\end{array}\right)=1
$$

Similarly, in a "dual" sense, we could have a visualization wherein the point $\mathrm{x}[0 \leq x \leq 0.5]$ divides the double-line in two parts of lengths $(0.5+x):(0.5-x):: \mathrm{O} 1$ to $\mathrm{x}: \mathrm{x}$ to $\mathrm{O} 3$.

Now, analogously-and-probabilistically, similar to [Sahai, 2009; 2006], the probability of ' $\mathrm{k}$ ' points/ knots being on the left of ' $0.5+x$ ', and ' $n-k$ ' points/ knots being on the right of ' $0.5-\mathrm{x}$ ' could easily be seen to be leading to the following equation. Here, the 'Dual Probability-Weights' " $P_{w 2}(x ; k, n)$ " are the typical coefficients of $\theta^{n}$ in the expansion of the binomial-product $\left[(1+\theta)^{n *(0.5+x)}\right] *\left[(1+\theta)^{n *(0.5-x)}\right]$, i.e. in $(1+\theta)^{n}$. Therefore, we have:

$$
\sum_{k=0}^{k=n} P_{w 2}(x ; k, n)=\sum_{k=0}^{k=n}\left(\begin{array}{c}
n *(0.5+x) \\
k
\end{array}\right) *\left(\begin{array}{c}
n *(0.5-x) \\
n-k
\end{array}\right)=1
$$

Apparently, both $P_{w 1}(x ; k, n)$ and $P_{w 2}(x ; k, n)$, dual to each-other, define a Probability Distribution each with $\mathrm{n}+1$ knots $\mathrm{k} /(2 * \mathrm{n})[\mathrm{k}=0(1) \mathrm{n}]$ being its support in $\mathrm{C}[0,0.5]$. As such, we define our Optimal Weights; Say $W_{\text {opt. }}(x ; k, n)$, through a simple Fusion of the two Probability Distributions above in (3) and (4) by taking their respective arithmetic mean. Therefore, we have:

$$
W_{\text {opt. }}(x ; k, n)=\frac{1}{2}\left[\left(\begin{array}{c}
n *(0.5-x) \\
k
\end{array}\right) *\left(\begin{array}{c}
n *(0.5+x) \\
n-k
\end{array}\right)+\left(\begin{array}{c}
n *(0.5+x) \\
k
\end{array}\right) *\left(\begin{array}{c}
n *(0.5-x) \\
n-k
\end{array}\right)\right]
$$

Thence, we propose a new approximation formula which uses the weight-functions derived using a probabilistic approach, and a rather-ingenious 'Fusion' of two dual perspectives, as above. This 'New Optimal Probabilistic Approximation Operator' happens to be as below.

$$
\left.O_{n}(f)\right)(x)=\sum_{k=1}^{k=n} w_{\text {opt. }}(x ; k, n) * f(k /(2 * n)
$$

Wherein, $W_{\text {opt. }}(x ; k, n)$ is as defined in (5). At this point, we note that the original interval $\mathrm{C}[0,1]$ is the domain of the impugned bounded function ' $\mathrm{f}(\mathrm{x})$ '. Also, we note that the interval $\left[\frac{1}{2}-\frac{x}{2}, \frac{1}{2}\right] \&\left[\frac{1}{2}, \frac{1}{2}+\frac{x}{2}\right]$ are the two sub-intervals of the domain. It is significant to observe that, with $x \in[0,1 / 2]$, whereas the former sub-interval will grow to become $\left[0, \frac{1}{2}\right]$ for $x=1 / 2$, the latter sub-interval will grow to become $\left[\frac{1}{2}, 1\right]$ for $x=1 / 2$. On the other hand, for $x=0$, both of them will degenerate to the point " $\frac{1}{2}$ "! In a sense, the two intervals are not only complimentary to each-other, but are also 'dual' to each-other. It is interesting to note that if we use $\mathrm{n}$ [ $\mathrm{n}$ being a positive integer] points/ knots beside zero on $\mathrm{C}[0$, $1 / 2]$, our proposed operator $\left(O_{n}(f)\right)(x)$ has Zero-Error for the Approximation of $f(x)=x^{m}$, for all $\mathrm{m} \epsilon[0, n]$, i. e. for all $\mathrm{m} \leq n$. Another point of curiosity would be to discover as to how our proposed operator $\left(O_{n}(f)\right)(x)$ performs vis-a-vis the well-known (Primal Version) Bersntein's Polynomial Approximation Operator. To accomplish that we have chosen FOUR example-functions in the following Empirical Study section, in the sense of simulation (assuming that the function $\mathrm{f}(\mathrm{x})$ is known). The aforesaid Empirical Simulation Study has been done using MAPLE 13. We have considered THREE illustrative values of $n$, namely $n=3,6$, and 9. The resultant values of the Percentage Relative Absolute Errors i.e. PRAbsErr (dot) are tabulated in the relevant FOUR Tables, correspondingly to each of the FOUR respective illustrative example-functions. 


\section{An Empirical Simulation Study}

This section is also of prime importance-and-interest, as herein we try to illustrate the potential of our proposed probabilistic operator " $\left(O_{n}(\mathrm{f})\right)(\mathrm{x})$ " . As rather-apparent in the preceding section, the proposed operator's mother-operator are the Bernstein's Polynomials for 'f (x)' namely, “( $\left.B_{n}(\mathrm{f})\right)(\mathrm{x})$ ” defined in (2). As such, we could not have any idea which could have been conclusive about the relative efficiency of the proposed estimator in terms of its better estimation potential algebraically. Hence, we have to discover and bring forth the same through our attempted "Empirical Simulation Study". In this study we have chosen FOUR illustrative example functions, namely: $\exp (x), 10^{x}, \sin (2+x), \& \ln (2+x)$. For the simplicity of the numerical illustration, we have confined to the chosen THREE ' $n$-values', namely: $n=3,6, \&$ 9. We have considered numerical values (per the illustrative numerical study described) of the "Percentage Relative Absolute Errors" using the relevant operators by the evaluation of the expressions: PRAbsError(.) (In $\%)$ for $\left(O_{n}(f)\right)(x) \&$ $\left(B_{n}(f)\right)(x)$, respectively :

$$
\frac{\left(\int_{0}^{0.5} O_{n}(f ; x) d x-\int_{0}^{0.5} f(x) d x\right)}{\int_{0}^{0.5} f(x) d x}=\operatorname{PRAbsErr}\left(O_{n}\right)
$$

And

$$
\frac{\left(\int_{0}^{0.5} B_{n}(f ; x) d x-\int_{0}^{0.5} f(x) d x\right)}{\int_{0}^{0.5} f(x) d x}=\operatorname{PRAbsErr}\left(B_{n}\right) .
$$

These aforesaid PRAbsError(.) (In\%) are calculated using the MAPLE 13 [Evaluation-Version Code ], and are tabulated in the following TABLES.The illustrations have amply justified-and-supported our proposed operator " $\left(O_{n}(\mathrm{f})\right)$ (x)" as they are performing exceedingly better than the " $\left(B_{n}(\mathrm{f})\right)(\mathrm{x})$ " defined in (2).

\section{References}

Cheney, E. W. and A. Sharma. (1964). Bernstein power series. Canad. J. Maths, 16, 241- 252.

Korovkin, P.P. (1960). Linear Operators and Approximation Theory. Hindustan Publishing, Delhi.

[Online] Available: http://www.maplesoft.com/contact/webforms/maple_evaluation.aspx. Maple 13 Evaluation-Version.

Sahai, Ashok and Govind Prasad. (1984). Sharp estimates of approximation by some positive linear operators. Bull. Australian Math. Soc., 29, 1984, pp. 13-18.

Sahai, Ashok \& Sameer Verma. (2009). Efficient quadrature operator using dual-perspectives-fusion probabilistic weights? IJET, 1(1), pp. 1-8.

Sahai, Ashok, Wahid, Shanaz A \& Madabhusi Raghunadh Acharya. (2009). Computerizable Iterative-Algorithmic Quadrature Operator Using an Efficient Two-Phase Modification of Bernstein Polynomial? IJET, 1(3), pp. $104-108$.

Sahai, Ashok, Shanaz A. Wahid \& Arvind Sinha. (2006). A positive linear operator using probabilistic approach? Journal of Applied Science, vol. 6, Issue 12, pp.2662-2665.

Sahai, Ashok. (2004). An iterative algorithm for improved approximation by Bernsteins operator using statistical perspective, Applied Mathematics and Comput., 149, pp. 327-335.

Weirstrass, K. (1885). Uber die analytische darstellbarkait sogenannter willkurlicher functionen einer reellen veranderlichen situngsberichteder koniglich preussischen akademie der wissenshcaften zu berlin, 633-639 \& 789-805.

\section{APPENDIX}

Table 3.1 Percentage Relative Absolute Error ["PRAbsErr (.)" (In \%)] of the Operators for Example-Function: $\mathrm{f}(\mathrm{x})=$ $\exp (\mathrm{x})$

\begin{tabular}{|c||c||c||c|}
\hline$\downarrow$ Operator & $\downarrow$ For $\mathbf{n}=\mathbf{3}$ & $\downarrow$ For $\mathbf{n}=\mathbf{6}$ & $\downarrow$ For $\mathbf{n}=\mathbf{9}$ \\
\hline$P R A b s E r r\left(O_{n}\right)$ & $\mathbf{0 . 0 0 0 9 5 8 1 3 1 0 6 1}$ & $\mathbf{0 . 0 0 0 0 1 5 4 1 4 9 4 1}$ & $\mathbf{0 . 0 0 0 0 0 1 9 8 8 5 2 7}$ \\
\hline$P R A b s E r r\left(B_{n}\right)$ & $\mathbf{1 3 . 1 7 7 8 3 8 9 9 0 0 0}$ & $\mathbf{1 2 . 8 1 0 0 3 9 3 4 0 0 0}$ & $\mathbf{1 2 . 6 8 5 8 7 7 8 5 0 0 0}$ \\
\hline
\end{tabular}

Table 3.2 Percentage Relative Absolute Error ["PRAbsErr (.)" (In \%)] of the Operators for Example-Function: $f(x)=10^{x}$

\begin{tabular}{|c||c||c||c|}
\hline$\downarrow$ Operator & $\downarrow$ For $\mathbf{n}=\mathbf{3}$ & $\downarrow$ For $\mathbf{n}=\mathbf{6}$ & $\downarrow$ For $\mathbf{n}=\mathbf{9}$ \\
\hline$P R A b s E r r\left(O_{n}\right)$ & $\mathbf{0 . 0 2 6 1 9 2 4 8 2 1 9 0}$ & $\mathbf{0 . 0 0 0 0 1 2 7 7 9 0 0 0}$ & $\mathbf{0 . 0 0 0 0 0 2 0 1 3 0 0 0}$ \\
\hline$P R A b s E r r\left(B_{n}\right)$ & $\mathbf{3 2 . 2 3 2 4 6 1 9 0 0 0 0}$ & $\mathbf{3 0 . 1 6 1 9 0 0 4 7 0 0 0}$ & $\mathbf{2 9 . 4 5 3 8 0 2 1 8 0 0 0}$ \\
\hline
\end{tabular}


Table 3.3 Percentage Relative Absolute Error ["PRAbsErr (.)" (In \%)] of the Operators for Example-Function: $f(x)=$ $\sin (2+x)$

\begin{tabular}{|c||c||c||c|}
\hline$\downarrow$ Operator & $\downarrow$ For $\mathbf{n}=\mathbf{3}$ & $\downarrow$ For $\mathbf{n}=\mathbf{6}$ & $\downarrow$ For $\mathbf{n}=\mathbf{9}$ \\
\hline$P R A b s E r r\left(O_{n}\right)$ & $\mathbf{0 . 0 0 0 9 7 0 9 1 7 2 1 4}$ & $\mathbf{0 . 0 0 0 0 1 0 3 8 9 6 9 7}$ & $\mathbf{0 . 0 0 0 0 0 1 7 9 2 2 2 2}$ \\
\hline$P R A b s E r r\left(B_{n}\right)$ & $\mathbf{1 0 . 8 0 0 2 8 4 3 6 0 0 0}$ & $\mathbf{1 0 . 4 6 9 6 7 4 3 7 0 0 0}$ & $\mathbf{1 0 . 3 6 0 7 4 9 6 7 0 0 0}$ \\
\hline
\end{tabular}

Table 3.4 Percentage Relative Absolute Error ["PRAbsErr (.)" (In \%)] of the Operators for Example-Function: $f(x)=$ $\ln (2+x)$

\begin{tabular}{|c||c||c||c|}
\hline$\downarrow$ Operator & $\downarrow$ For $\mathbf{n}=\mathbf{3}$ & $\downarrow$ For $\mathbf{n}=\mathbf{6}$ & $\downarrow$ For $\mathbf{n}=\mathbf{9}$ \\
\hline$P R A b s E r r\left(O_{n}\right)$ & $\mathbf{0 . 0 0 0 2 8 3 0 1 3 8 8 0}$ & $\mathbf{0 . 0 0 0 0 7 9 1 2 3 2 2 0}$ & $\mathbf{0 . 0 0 0 0 0 2 7 6 9 3 1 0}$ \\
\hline$P R A b s E r r\left(B_{n}\right)$ & $\mathbf{6 . 7 2 2 6 0 0 1 9 1 0 0 0}$ & $\mathbf{6 . 8 0 3 3 9 4 9 1 6 0 0 0}$ & $\mathbf{6 . 8 2 9 9 3 7 0 7 3 0 0 0}$ \\
\hline
\end{tabular}

